\title{
Sensitivity of KM3NeT/ARCA to the neutrino flavour composition
}

Thomas Eberl*

Friedrich Alexander Universität Erlangen-Nürnberg, Erlangen Centre for Astroparticle Physics, Erwin-Rommel-Str. 1, 91058 Erlangen, Germany

E-mail: Thomas.Eberlefau.de

Thomas Heid

Friedrich Alexander Universität Erlangen-Nürnberg, Erlangen Centre for Astroparticle Physics, Erwin-Rommel-Str. 1, 91058 Erlangen, Germany

E-mail: Thomas.Heidefau.de

\section{on behalf of the KM3NeT Collaboration}

KM3NeT is a distributed neutrino research infrastructure in the Mediterranean Sea. KM3NeT/ARCA is a high-energy neutrino telescope, dedicated to the search for extraterrestrial neutrino sources in the TeV-PeV range. One major goal is the identification of the sources of the neutrino flux recently discovered by IceCube. Furthermore, KM3NeT/ARCA is optimised to study Galactic neutrino point sources. The analysis of the flavour composition of astrophysical neutrinos arriving at Earth can shed light on the production mechanisms of these neutrinos inside their astrophysical sources. The distinction between different neutrino flavours is only possible on a statistical basis and a method called "spectral fitting" is employed to this end. In order to estimate the sensitivity of KM3NeT/ARCA to the flavour composition using this method, spectra obtained from Monte-Carlo-based pseudo-data samples are compared to expectations from neutrino flux models for signal and background. To increase the power of the spectral fitting procedure, the event sample is separated into multiple subsamples according to their event type. Therefore, an artificial neural network is used to discriminate between five target types: double bang events, cascades and three different track-like event types.

35th International Cosmic Ray Conference - ICRC2017

10-20 July, 2017

Bexco, Busan, Korea

${ }^{*}$ Speaker. 


\section{Introduction}

KM3NeT is a distributed neutrino infrastructure in the Mediterranean Sea. It consists of a network of deep-sea neutrino telescopes whose design is optimized to detect neutrinos in different energy ranges. KM3NeT/ARCA, located at a distance of about $100 \mathrm{~km}$ from the Eastern Sicilian coast, at about $3500 \mathrm{~m}$ under the sea level, will study high-energy cosmic neutrinos. ARCA will consist of two building blocks, each equipped with 115 Detection Units (DU) which are separated on average by $90 \mathrm{~m}$. Each DU contains 18 Digital Optical Modules (DOM) vertically separated by $36 \mathrm{~m}$. A DOM is equipped with 313 inch photomultiplier tubes resulting in a close-to $4 \pi$-acceptance. The total instrumented volume is around $1 \mathrm{~km}^{3}$.

First measurements of a cosmic neutrino flux have been established in the last years. However, measurements of the ratio between the different neutrino flavours are not conclusive up to now. Firstly, the neutrino flavour composition at the source is related to the mechanisms acting at the neutrino production site. Then, also neutrino oscillations play a significant role in modifying the flavour ratio of the signal. Furthermore, exotic phenomena, such as the presence of sterile neutrinos or non-standard interactions in matter, can change the flavour composition at several stages. Since the discovery of the diffuse neutrino flux with IceCube, multiple analyses of the flavour composition were published. The ratio between recognised shower-like and track-like events was used to investigate the signal flavour composition [1] and to constrain even non-standard physics scenarios such as flavour decay [1] [2]. Further analyses followed the publication and the release of new event samples by IceCube [3] [4] [5]. The first results on the flavour composition published by the IceCube collaboration were based on the so-called High Energy Starting Event (HESE) sample. [6]. In that work, most of the flavour composition space phase could not be statistically excluded. In more recent analyses, multiple neutrino samples are combined into one likelihood-fitting procedure [7] [8]. Due to the extended livetime of the used data and because of a better selection of the neutrino samples, a larger statistics of neutrino candidates could be used in the analysis. Consequently, the allowed parameter space at given confidence level could be constrained such that a flux consisting only of electron neutrinos at the source could be excluded with a significance of $3.7 \sigma$. The present best fit suggests an equal amount of $v_{\mathrm{e}}$ and $v_{\mu}$.

In this work, the flavour composition is evaluated by using pseudo-data sets and by categorizing the identified events into different event types. In this context, the excellent direction and energy resolutions of the

KM3NeT/ARCA detector permit to select large and relatively pure samples of a given event type. In the analysis, expectations from neutrino flux models are fitted to the pseudo-data that mimicks the measurement. Each of these steps will be explained in the following sections.

\section{Analysis}

Samples of dedicated neutrino simulations are available, representing all neutrino flavours and interaction channels. Events can be weighted according to atmospheric and astrophysical neutrino flux models. The former is chosen as the Honda et al. [9] computations for its conventional component, while the Enberg et al. model [10] is used for the prompt contributions, corrected to 
account for the cosmic ray (CR) knee [11]. The latter is parametrised for each flavour $l$ as:

$$
\Phi_{l}=\Phi_{0 l} \cdot 10^{-18}\left(\frac{E}{100 \mathrm{TeV}}\right)^{-\gamma}
$$

where $\Phi_{0}$ is the normalisation of the flux, $\gamma$ is the spectral index and $E$ is the energy of the neutrino. The same spectral index is assumed for each neutrino flavour spectrum. The normalisation and spectral shape are not fixed, as these values will be fitted in the analysis. Other possibilities, such as multiple power-law or spectral cut-offs are not considered in this work.

Down-going atmospheric muons are simulated to study the muon background. Here the parametrised simulation with the MUPAGE package [12] [13] is used. The following steps in the simulation of the detector response take care of light and particle production and propagation, as well as of the photon detection with the DOM and the digitization by the data acquisition system.

\subsection{Event identification}

The spectral fitting procedure is performed on multiple different spectra simultaneously. These spectra have to be statistically independent of each other, i.e. no event is present in more than one subsample. Event identification algorithms are used to separate the complete sample into several subsamples with consistent event type. Artificial neural network classifiers show a good performance on this task. An artificial neural network is a machine learning algorithm which classifies events into different predefined types. This is achieved by training the network on so-called features, i.e. observables characterising the neutrino interaction. These observables are inputs to the classifier and consist of the results of several different reconstruction algorithms. Further dedicated features are added to collect more information about the type and geometrical shape of each event. A principle component analysis of the feature space is performed beforehand, in order to reduce its dimensions and to transform the features into linearly uncorrelated quantities. The events are weighted in such a way that the size of the different event type samples or classes is balanced for a given neutrino energy.

The employed neural network is based on the python package theanets [14]. The so-called hyper-parameters defining the layout of the neural network are optimally found with a random search within given ranges. The used neural network is a shallow network type with one hidden layer. The hidden layer consists of 46 neurons. The input layer is built up with as many neurons as there are features available. The output layer consists of 5 output units. Each neuron of the output layer returns a value called safety, which can be interpreted as the probability that the considered event belongs to a given class.

Five output classes are defined, which are used independently for the spectral fitting:

1. track events: events with outgoing muon. These are charged-current $v_{\mu}$ or charged-current $v_{\tau}$ events, in which the $\tau$ decays to a muon. Tracks are further subdivided into:

(a) down-going tracks starting outside the instrumented volume

(b) starting tracks events of arbitrary direction, i.e. tracks originating inside the instrumented volume

(c) up-going tracks starting outside the instrumented volume 
2. cascades: all neutrino interactions via the neutral-current and $\tau$ decays closer than $13 \mathrm{~m}$ to the initial vertex are also labeled as cascades

3. double bang events: $v_{\tau}$ interacting in the charged-current channel, where the $\tau$ decays into a shower after a distance larger than $13 \mathrm{~m}$

The training-data sample for the network includes mostly events that are well reconstructed either by the track or by the cascade reconstruction algorithm. A lower threshold on the reconstructed energy of $1 \mathrm{TeV}$ is applied. The cascade vertex must be reconstructed in a fiducial volume around the detector and the track fit quality has to overcome a loose threshold.

The first three classes are dominated by atmospheric muons. However, the muon contamination dominates also the neutrino contribution by one or two orders of magnitude in the other classes and a further atmospheric muon rejection is necessary. A rejection of atmospheric muons down to a percent-level of residual contamination of the neutrino samples is achieved by cutting on various class-individual and appropriately chosen quantities that are mainly based on spatial and temporal distributions of the detected photons.

Finally, the event rates, as number of detected events per year, shown in Figure 1 are obtained. Residual atmospheric muons with a negligibly small rate are expected, if at all, only for energies larger than $10^{5} \mathrm{GeV}$
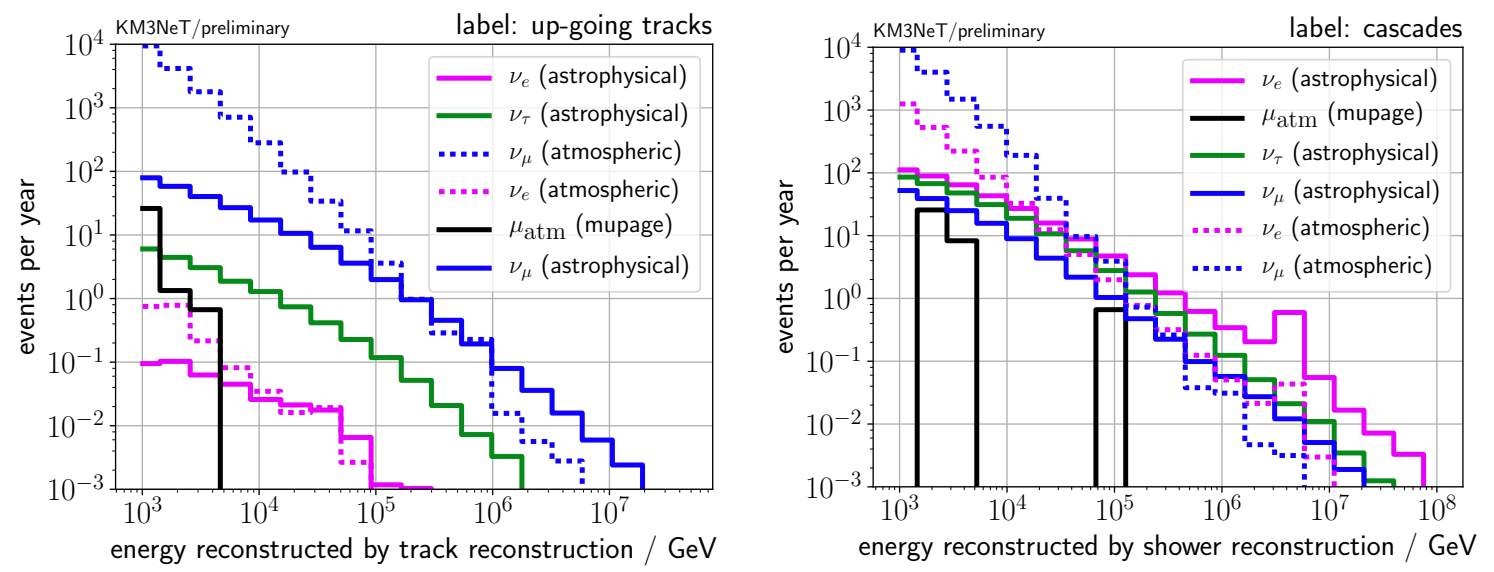

Figure 1: Flux of classified events in KM3NeT/ARCA. Left: events classified as up-going tracks. Right: events classified as cascades.

Only up-going track-like and cascade-like events are used in this analysis. Figure 1 shows the expectations for one possible measurement. The simulation and determination of the sensitivity to the flavour composition starts from the obtained input distributions.

\subsection{The spectral fit}

The first step of the spectral fitting procedure is the generation of pseudo-experiments. After fixing the strength of each contribution, the number of events per bin contributing to one component is drawn from a Poissonian distribution. All components are added to result in a simulated spectrum corresponding to one possible experimental measurement with KM3NeT/ARCA. As the strength of 
each astrophysical flavour component is not known a priori, the composition can change from one pseudo-experiment to another.

The main step of the spectral fitting procedure is the fit itself. Each bin of the distributions will be populated by a few (if not zero) events. A Poissonian likelihood fit is then used. The fit uses the sherpa package [15], developed in the domain of $\mathrm{x}$-ray astronomy. The minimisation uses a Levenberg algorithm [16].

The first interesting quantity to be fitted is the normalisation factor of the cosmic signal for each neutrino flavour. The atmospheric neutrino flux is the most important remaining background for the measurement. As the systematic uncertainty on its normalisation is rather large (of the order of $25 \%$ ), it is also fitted. It has been verified that different true values of the atmospheric neutrino flux normalisation then have only a negligible remaining influence on the results of the spectral fitting procedure.

The spectral index of the astrophysical flux is not known very well up to now. Hence, also this quantity must be left free in the fit, which results in an overall worsening of the flavour composition sensitivity. Tests have shown that fixing the spectral index to very hard or very soft values, the spectral fit has a strong tendency to over- or underestimate the $v_{\tau}$ contribution. Fitting the spectral index together with the normalisation of the astrophysical flux and the background normalisation successfully avoids this behaviour of the fit.

\section{Results}

A large number of pseudo-experiments has been run and analysed, following the methodologies described above, while the envisaged measurement will result in exactly one best-fit value. Starting with this value, confidence and significance levels are computed. To test the behaviour of the procedure, pseudo-experiments with an equipartition of the three neutrino flavours as true value are used. Furthermore, the pseudo-experiments are binned in flavour composition space. Sorting the binned results according to their frequency, the significance and confidence levels in flavour composition space can be computed. This is presented in figure 2 . The resulting values are shown, together with the significance of the rejection of such a flavour composition.

Figure 2 shows the significance levels, if an experiment would result in the outcome of equipartition in the three neutrino flavours. A symmetrical shape around the expected value is observed if the fit is only performed on the normalisation of the individual neutrino fluxes. The elongated shape of the ellipse is due to the smaller separation power between the $v_{\tau}$ and $v_{\mathrm{e}}$ with respect to the $v_{\mu}$ content. Therefore, the fraction of $v_{\mu}$ is given in a range between 0.3 and 0.4. However, the ratio of the other two neutrino flavours can vary by a larger amount. The tilt of the shape with respect to the $v_{\mu}$ axis results from the $v_{\tau}$ with a muon leaving the tau decay vertex.

The right plot of figure 2 shows the obtained significance levels when also the spectral index of the astrophysical flux is fitted. The shape broadens because of the addition of more nuisance parameters in the fit. The overall shape, tilt and elongation, remains the same. However, the ellipse is no longer centered around the expected value, but shifted towards the $v_{\mu}$-axis. Experiments with a given truth are usually fitted with a too high $v_{\tau}$ contribution.

A pseudo-experiment assuming a true equipartition in the three neutrino flavours and a true spectral index $\gamma=2.3$ has been performed. Its outcome is reported in figure 3. Here the distribution 

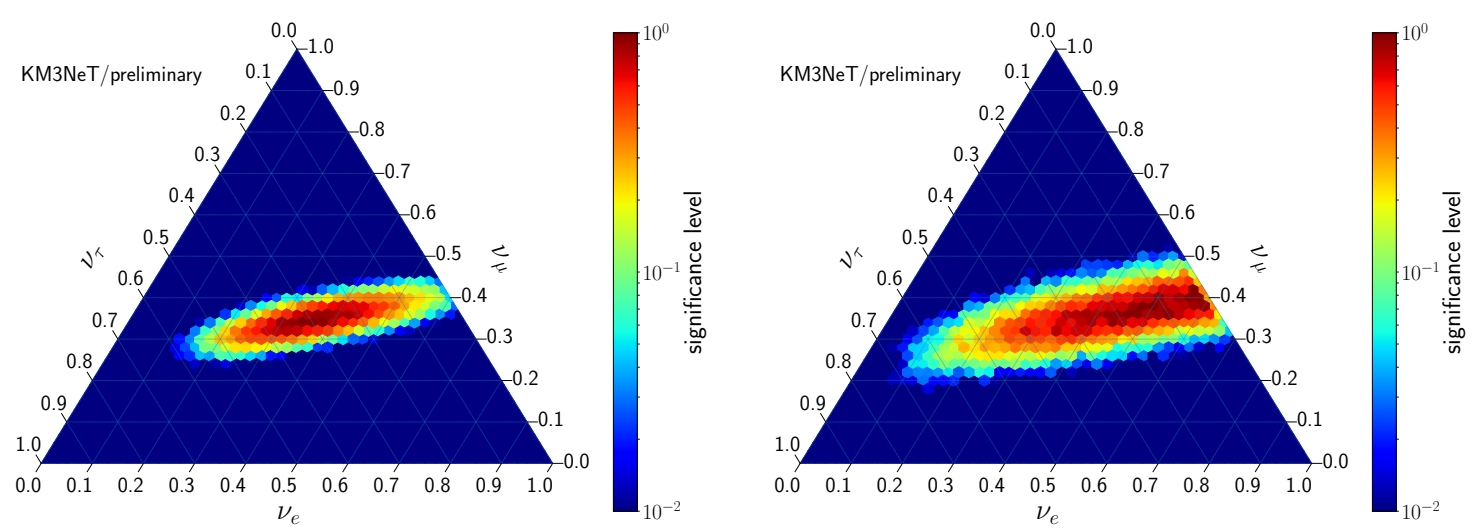

Figure 2: The significance level of an experiment with the outcome of of equipartition in the three neutrino flavours. $O\left(10^{8}\right)$ pseudo-experiments were generate uniformly in flavour composition space. The livetime of KM3NeT/ARCA was fixed to 10 years. Left: only the astrophysical neutrino flux normalisation is fitted. Right: the normalisation of the astrophysical and atmospherical neutrino flux and the spectral index of the astrophysical flux are fitted. Due to the limited number of pseudo-experiments, the lower limit of the significance for this evaluation of the KM3NeT/ARCA performance is $10^{-2}$. Lower values are rounded to that value.

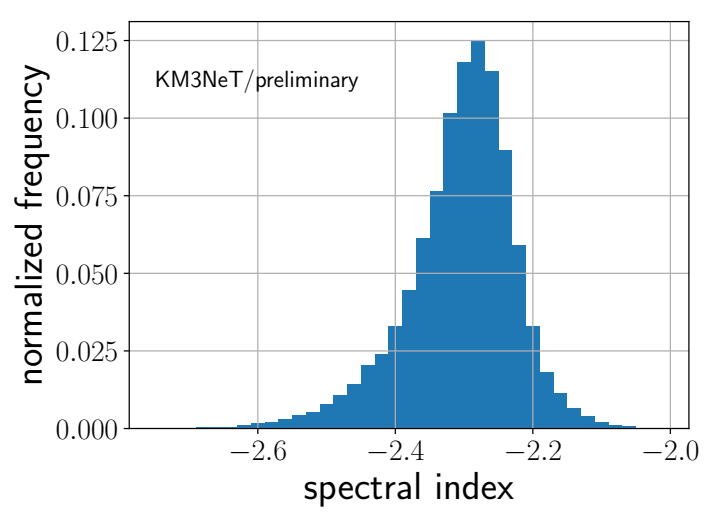

Figure 3: Distribution of the fitted spectral index in full fit mode. The truth value was -2.3. Experimental outcomes for the true flavour ratio value $1: 1: 1$ are shown.

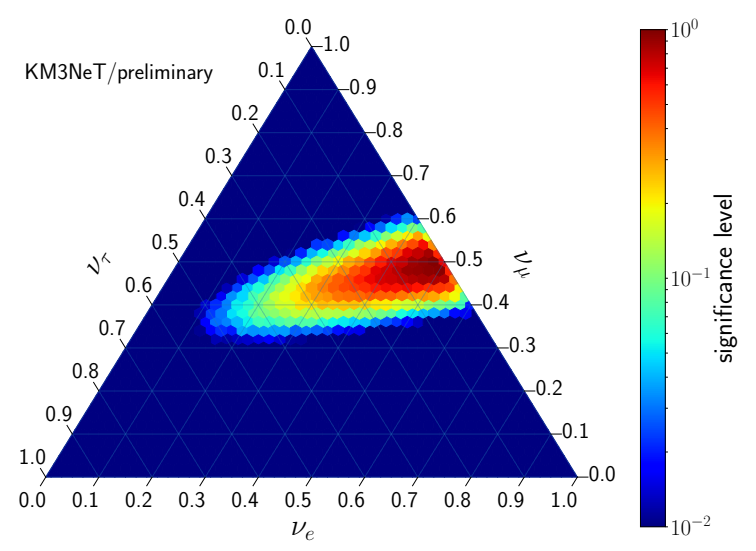

Figure 4: The significance levels of an experiment with a outcome of 1:1:0 for $v_{\mathrm{e}}: v_{\mu}: v_{\tau} . O\left(10^{8}\right)$ pseudo experiments were generated uniformly in flavour composition space. Lifetime was fixed to 10 years of ARCA measurements. The normalisation of the astrophysical and atmospherical neutrino flux and the spectral index of the astrophysical flux are fitted. Due to the limited number of pseudo-experiments, the lower limit of the significance for this evaluation of the KM3NeT/ARCA performance is $10^{-2}$. Lower values are rounded to that value.

of the fitted spectral index of the astrophysical flux is reported, and it is well-centred around the truth value.

Finally, Figure 4 shows the significance levels that result, when measuring an equal $v_{\mu}$ and $v_{\mathrm{e}}$ 
contribution, in absence of $v_{\tau}$. This actually corresponds to the best fit in the IceCube analysis, even though this result is not yet statistically significant. In these pseudo-experiments the best fit position is shifted towards the experimental outcome, with the same broad elliptical shape.

\section{Conclusion}

We have developed a method to fit the neutrino flavour composition with KM3NeT/ARCA. Here, two samples of events are considered: cascades and up-going tracks. Future analyses could benefit from additional event topologies, such as the tau-double-bang signature. In this analysis the fit is performed on spectra using the reconstructed energies. Quantities providing a higher sensitivity might exist. The atmospheric muon flux is neglected in the fitting procedure due to the very low number of expected events after the muon rejection cuts. Besides the normalisation and spectral index of the astrophysical neutrino flux, also the normalisation of the atmospheric neutrino flux has been fitted. After ten years of data taking with ARCA, a wide range of the flavour composition space can be rejected on a 95\% significance level. The spectral index can also be fitted to good precision.

\section{References}

[1] O. Mena, S. Palomares-Ruiz, and A. C. Vincent, Flavor composition of the high-energy neutrino events in IceCube, Phys. Rev. Lett. 113 (Aug, 2014) 091103.

[2] G. Pagliaroli, A. Palladino, F. L. Villante, and F. Vissani, Testing nonradiative neutrino decay scenarios with IceCube data, Phys. Rev. D 92 (Dec, 2015) 113008.

[3] A. Palladino, G. Pagliaroli, F. L. Villante, and F. Vissani, What is the Flavor of the Cosmic Neutrinos Seen by IceCube?, Phys. Rev. Lett. 114 (2015), no. 17171101.

[4] S. Palomares-Ruiz, O. Mena, and A. C. Vincent, On the flavor composition of the high-energy neutrinos in IceCube, Nuclear and Particle Physics Proceedings 273 (2016) 433 - 439.

[5] S. Palomares-Ruiz, A. C. Vincent, and O. Mena, Spectral analysis of the high-energy IceCube neutrinos, Phys. Rev. D 91 (May, 2015) 103008.

[6] IceCube Collaboration, Flavor ratio of astrophysical neutrinos above 35 tev in IceCube, Phys. Rev. Lett. 114 (Apr, 2015) 171102.

[7] IceCube Collaboration, A combined maximum-likelihood analysis of the high-energy astrophysical neutrino flux measured with IceCube, The Astrophysical Journal $\mathbf{8 0 9}$ (2015), no. 198 .

[8] L. Mohrmann, Characterization of the astrophysical neutrino flux at the IceCube neutrino observatory, Journal of Physics: Conference Series 718 (2016), no. 6062045.

[9] M. Honda, T. Kajita, K. Kasahara, S. Midorikawa, and T. Sanuki, Calculation of atmospheric neutrino flux using the interaction model calibrated with atmospheric muon data, Phys. Rev. D 75 (Feb, 2007) 043006. 
[10] R. Enberg, M. H. Reno, and I. Sarcevic, Prompt neutrino fluxes from atmospheric charm, Phys. Rev. D 78 (Aug, 2008) 043005.

[11] IceCube Collaboration, Search for a diffuse flux of astrophysical muon neutrinos with the IceCube 59-string configuration, Phys. Rev. D 89 (Mar, 2014) 062007.

[12] G. Carminati, M. Bazzotti, A. Margiotta, and M. Spurio, Atmospheric muons from parametric formulas: a fast generator for neutrino telescopes (MUPAGE), Computer Physics Communications 179 (2008), no. 12915 - 923.

[13] Y. Becherini, A. Margiotta, M. Sioli, and M. Spurio, A parameterisation of single and multiple muons in the deep water or ice, Astroparticle Physics 25 (2006), no. 11 - 13.

[14] L. Johnson, "theanets v0.7.3." https://github.com/lmjohns3/theanets.

[15] P. Freeman, S. Doe, and A. Siemiginowska, Sherpa: a mission-independent data analysis application, in Astronomical Data Analysis (J.-L. Starck and F. D. Murtagh, eds.), vol. 4477 of procspie, pp. 76-87, Nov., 2001.

[16] J. More, Levenberg-Marquardt algorithm: implementation and theory. Jan, 1977. 